\title{
SOLUTIONS OF FRACTIONAL DIFFUSION EQUATIONS AND CATTANEO-HRISTOV DIFFUSION MODEL
}

\author{
NDOLANE SENE* \\ ${ }^{1}$ Laboratoire Lmdan, Département de Mathématiques de la Décision, Université Cheikh Anta Diop de \\ Dakar, Faculté des Sciences Economiques et Gestion, BP 5683 Dakar Fann, Senegal
}

*Corresponding author: ndolanesene@yahoo.fr, ndolane.sene@ucad.edu.sn

\begin{abstract}
The analytical solutions of the fractional diffusion equations in one and two-dimensional spaces have been proposed. The analytical solution of the Cattaneo-Hristov diffusion model with the particular boundary conditions has been suggested. In general, the numerical methods have been used to solve the fractional diffusion equations and the Cattaneo-Hristov diffusion model. The Laplace and the Fourier sine transforms have been used to get the analytical solutions. The analytical solutions of the classical diffusion equations and the Cattaneo-Hristov diffusion model obtained when the order of the fractional derivative converges to 1 have been recalled. The graphical representations of the analytical solutions of the fractional diffusion equations and the Cattaneo-Hristov diffusion model have been provided.
\end{abstract}

\section{INTRODUCTION}

In fractional calculus, we have many fractional derivatives operators as: the Riemann-Liouville fractional derivative [34] [36], the Caputo fractional derivative [8] [41], the Atangana-Baleanu fractional derivative [2] [3] [4], the Caputo-Fabrizio fractional derivative [6] [30], the Conformable fractional derivative [42], the generalized fractional derivatives in Caputo and Riemann-Liouville sense [21] [22] [24], and others. Fractional calculus has many applications in mechanic, physics and science and engineering. Fractional calculus has many applications in the viscoelastic models and the diffusion models. In [12] [13], Hristov treats on heat

Received 2018-12-03; accepted 2019-01-09; published 2019-03-01.

2010 Mathematics Subject Classification. 42A38, 76R50, 26A33 .

Key words and phrases. Fractional diffusion equation; Caputo fractional derivative; Cattaneo-Hristov diffusion model.

(c)2019 Authors retain the copyrights of their papers, and all open access articles are distributed under the terms of the Creative Commons Attribution License. 
diffusion equation in term of the Caputo-Fabrizio time fractional derivative. In [10], Hristov proposes new equations related to the fractional diffusion equations using the Atangana-Baleanu fractional derivative, see others models in [23]. In [1], Alkahtani and Atangana discuss the numerical solution of the Cattaneo-Hristov diffusion equation. In [26] Koca et al. propose the numerical solution of the second term of the CattaneoHristov diffusion equation. In [27], Li et al. have studied the Cauchy problem for nonlinear fractional time-space generalized Keller-Segel equation using the Caputo fractional derivative. In [29], Yranli et al. devoted to comparing the smoothing performance between the time fractional diffusion equation and the classical diffusion equation using the regulation method, Savitzky-Golay, and coverer method. In [37], Ruan et al. study a simultaneous identification problem of piecewise source term and the fractional order for time-fractional diffusion equation. In [45], Zhang and al. propose a discrete form for solving time fractional convection-diffusion equation. In [31], Ma et al. study asymptotic of the solutions to the fractional anomalous diffusion equations. Several works related to the fractional diffusion equations exist in the literature. The papers [5] [33] [39] [44] treat on fractional diffusion equations.

The fractional diffusion equation is obtained when a specific fractional derivative operator replaces the ordinary derivative in the classical diffusion equation. In this paper, we use the Caputo fractional derivative. Podlubny [35] has introduced the fractional diffusion equation in fractional calculus. We propose the analytical solutions of the fractional diffusion equations in one and two-dimensional spaces. Hristov [12] introduced the Cattaneo-Hristov diffusion equation in fractional calculus. The author [12] opens news problems related to the analytical or approximate solutions of the Cattaneo-Hristov diffusion equation. Koca et al. [26] propose the numerical and analytical solutions of the elastic part of the heat diffusion equation process. Alkahtani et al. [1] propose the numerical solution of the complete Cattaneo-Hristov diffusion equation using the Crank-Nicholson numerical scheme. Hristov [12] proposes an approximate solution of the Cattaneo-Hristov diffusion equation using the heat-balance integral method (HBIM). Hristov [12] proposes a double integral-balance method (DIM) to get the approximate solution of the Cattaneo-Hristov diffusion equation. The analytical or approximate solutions of the fractional diffusion equations using HBIM and DIM were proposed in [14] [15] [16] [17] [18] [19] [20] [32]. The analytical solution of the Cattaneo-Hristov equation was stated in [26] by Koca et al. They give the analytical solution of the elastic part of the heat diffusion equation process. In this paper, we continue the work concerning the analytical solution stated by Koca et al. in [26]. In this paper, we propose the analytical solution of the complete Cattaneo-Hristov diffusion equation of the transient heat equation using an integral method. The integral method uses both the Fourier sine transform and the Laplace transform. We will notice this integration method will permit to express the analytical solutions of the fractional diffusion equations in the term of the Gaussian error function and the Mittag-Leffler function [9] [40]. The graphical representations of the analytical solutions of some particular fractional diffusion equations are provided. 
The paper is organized as follows: in Section 2, we recall preliminary definitions which we will use in this paper. In Section 3, we analyze the analytical solutions of the fractional diffusion equation in one-dimensional space. In Section 4, we get the analytical solution of the fractional diffusion equation in two-dimensional space. In Section 5, we analyze some particular cases graphically. And we finish with Section 6 by giving the conclusions and remarks.

\section{Fractional diffusion equations}

In this section, we present the fractional differential equations studied in this paper. The problems concern the fractional diffusion equation in one and two-dimensional spaces. The classical diffusion equation defined by the ordinary derivative is popular. Many works related to the analytical and the numerical solutions exist. Diffusion phenomena, of heat or mass [12] [35] is represented as the following form

$$
\frac{\partial u(x, t)}{\partial t}=\kappa^{2} \frac{\partial^{2} u(x, t)}{\partial x^{2}}
$$

where $\kappa^{2}=\frac{K}{\rho C_{p}}$. We add the following informations.

- $K$ represents the thermal conductivity,

- $\rho$ represents the specific heat,

- $C_{p}$ represents the density of the material,

- $-u$ represents the temperature distribution of the material.

The fractional diffusion equation is obtained when we replace the ordinary derivative by a fractional derivative operators. The fractional diffusion equation described by the Caputo fractional derivative is expressed in one-dimensional space by the following equation [11] [31] [35]

$$
D_{\alpha}^{c} u(x, t)=\kappa^{2} \frac{\partial^{2} u(x, t)}{\partial x^{2}}
$$

where $D_{\alpha}^{c}$ represents the Caputo fractional derivative operator defined by [28] [40]

$$
D_{\alpha}^{c} u(x, t)=\frac{1}{\Gamma(1-\alpha)} \int_{0}^{t} \frac{u^{\prime}(x, s)}{(t-s)^{\alpha}} d s
$$

all $t>0, \alpha \in(0,1)$, and $\Gamma($.$) denotes the Gamma function. \kappa^{2}$ represents the diffusion coefficient for the density of the diffusion material. The boundary conditions considered in this paper are the Dirichlet boundary conditions:

$$
\begin{aligned}
& \text { - }-u(x, 0)=0 \text { for } x>0, \\
& \text { - }-u(0, t)=1 \text { for } t>0
\end{aligned}
$$

The fractional diffusion equation exists in two dimensional space. It is expressed as the following form [31]

$$
D_{\alpha}^{c} u(x, y, t)=\kappa^{2}\left\{\frac{\partial^{2} u(x, y, t)}{\partial x^{2}}+\frac{\partial^{2} u(x, y, t)}{\partial y^{2}}\right\}
$$

with the Dirichlet boundary conditions defined as 
- $\bullet(x, y, 0)=0$ for $x, y>0$,

$\bullet \bullet u(0, y, t)=u(x, 0, t)=1$ for $t>0$.

The initial boundary conditions play an important role in the integral method. Note that, when the boundary conditions change, the form of the analytical solutions changes also. There exist many methods to get the analytical solutions of the fractional diffusion equations: as the Laplace transform, as the Fourier sine transform [43], as the heat-balance integral method (HBIM) [18,20], as a double integral method (DIM) [18,20], as a multiple integral method (MIM) [14]. This paper proposes an integral method consisting of applying both the Laplace transform and the Fourier sine transform. Let recall the Laplace transform of the Caputo fractional derivative which we will use later [25] [38]

$$
\mathcal{L}\left\{D_{\alpha}^{c} f(t)\right\}=s^{\alpha} \mathcal{L}\{f(t)\}(s)-s^{\alpha-1} f(0)
$$

where $\alpha \in(0,1)$. The transformation $(2.5)$ is known very useful in the resolution of the fractional differential equations. All solutions obtained in this paper will be rewritten using the Mittag-Leffler function [9] defined as the following form

$$
E_{\alpha, \beta}(z)=\sum_{k=0}^{\infty} \frac{z^{k}}{\Gamma(\alpha k+\beta)} .
$$

where $\alpha>0, \beta \in \mathbb{R}$ and $z \in \mathbb{C}$. We obtain the exponential function when $\alpha=\beta=1$ and we obtain the Mittag-Leffler function with one parameter when $\beta=1$, for more information see in [9].

\section{Analytical Solution of Fractional Diffusion Equation in One Dimensional space}

In this section, we investigate to find the analytical solution of the fractional diffusion equation in onedimensional space defined as the following form

$$
D_{\alpha}^{c} u(x, t)=\kappa^{2} \frac{\partial^{2} u(x, t)}{\partial x^{2}} .
$$

We consider the Dirichlet boundary conditions defined as follows:

$$
\begin{aligned}
& \bullet \bullet u(x, 0)=0 \text { for } x>0, \\
& \bullet \bullet-u(0, t)=1 \text { for } t>0 .
\end{aligned}
$$

In other words, we assume that the initial temperature of the material is null and the temperature of the plate for all $t>0$ is maintained constant $U_{0}=1$. It is essential for our results and the application of our method of resolution. The integral methods as HBIM and DIM use the finite penetration depth to get the approximate solutions of the fractional diffusion equation (3.1). For more information on these integral methods, see in [18] [20]. In this paper, we adopt the following integral method (see in [43]), described as follows:

- • apply the Fourier sine transform,

- • apply the Laplace transform, 


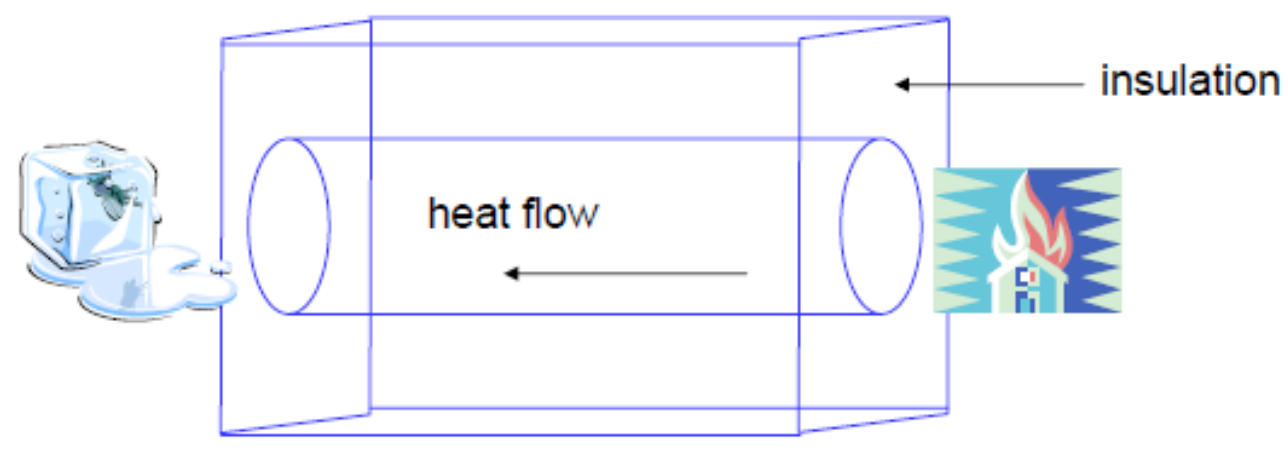

Figure 1. Fractional diffusion model.

- - apply the inverse of Laplace transform,

- $\bullet$ apply the inverse of Fourier sine transform.

This method of resolution seems very useful and practical to get the analytical solution of the fractional diffusion equations. If the temperature of the plate is null, the integral method described above seems no adequate to be applied and it is better to use the classical methods as HBIM or DIM to solve equation (3.1).

We begin the resolution of the fractional differential equation (3.1) by applying the Fourier sine transform. Multiplying equation (3.1) by $\frac{2}{\pi} \sin w x$ and integrating it between 0 to $\infty$, we get that:

$$
\begin{aligned}
& D_{\alpha}^{c} u_{s}(w, t)=\kappa^{2}\left\{\frac{2}{\pi} w u_{s}(0, t)-w^{2} u_{s}(w, t)\right\} \\
& D_{\alpha}^{c} u_{s}(w, t)=\frac{2 \kappa^{2} w}{\pi}-\kappa^{2} w^{2} u_{s}(w, t) .
\end{aligned}
$$

where $u_{s}(w, t)$ denotes the Fourier sine transform of $u(x, t)$. Rearranging, we obtain the following fractional differential equation defined as

$$
D_{\alpha}^{c} u_{s}(w, t)+\kappa^{2} w^{2} u_{s}(w, t)=\frac{2 \kappa^{2} w}{\pi} .
$$

The second step of the resolution consists of applying the Laplace transform to both sides of equation (3.2), and then we obtain that

$$
\begin{aligned}
s^{\alpha} \bar{u}_{s}(w, s)+\kappa^{2} w^{2} \bar{u}_{s}(w, s) & =\frac{2 \kappa^{2} w}{\pi s} \\
\bar{u}_{s}(w, s) & =\frac{2 \kappa^{2} w}{\pi s\left(s^{\alpha}+\kappa^{2} w^{2}\right)} .
\end{aligned}
$$

where $\bar{u}_{s}(w, s)$ denotes the Laplace transform of $u_{s}(w, t)$. The third step of the resolution consists of applying the inverse of the Laplace transform to both sides of equation (3.3). To reach our end, we rewrite equation (3.3) as follows:

$$
\bar{u}_{s}(w, s)=\frac{2}{\pi}\left\{\frac{1}{s}-\frac{s^{\alpha-1}}{s^{\alpha}+\kappa^{2} w^{2}}\right\} \frac{1}{w} .
$$


Applying the inverse of Laplace transform to both sides of equation (3.4) and using the Mittag-Leffler function as defined in [9], we get

$$
u_{s}(w, t)=\frac{2}{\pi w}\left\{1-E_{\alpha}\left(-\kappa^{2} w^{2} t^{\alpha}\right)\right\} .
$$

To get the analytical solution of the fractional diffusion equation (3.1), we apply the inverse of the Fourier sine transform to both sides of equation (3.5), and then we obtain the following result

$$
\begin{aligned}
u(x, t) & =\frac{2}{\pi} \int_{0}^{\infty} \frac{\sin w x}{w}\left\{1-E_{\alpha}\left(-\kappa^{2} w^{2} t^{\alpha}\right)\right\} d w \\
& =1-\frac{2}{\pi} \int_{0}^{\infty} \frac{\sin w x}{w} E_{\alpha}\left(-\kappa^{2} w^{2} t^{\alpha}\right) d w
\end{aligned}
$$

Let now analyze a particular case of the fractional diffusion equation. The classical diffusion equation is obtained when $\alpha \rightarrow 1$. To get the analytical solution, we use the Laplace transform obtained in equation (3.3). We have the following decomposition

$$
\bar{u}_{s}(w, s)=\frac{2}{\pi}\left\{\frac{1}{s}-\frac{1}{s+\kappa^{2} w^{2}}\right\} \frac{1}{w} .
$$

Using the inverse of Laplace transform to both sides of equation (3.7), we get the following intermediary solution

$$
u_{s}(w, t)=\frac{2}{\pi w}\left\{1-\exp \left(-\kappa^{2} w^{2} t\right)\right\} .
$$

Respecting the procedure of the resolution, we have to apply the inverse of Fourier sine transform, and then we obtain the analytical solution of the classical diffusion equation given by

$$
\begin{aligned}
u(x, t) & =\frac{2}{\pi} \int_{0}^{\infty} \frac{\sin w x}{w}\left\{1-\exp \left(-\kappa^{2} w^{2} t\right)\right\} d w \\
& =1-\frac{2}{\pi} \int_{0}^{\infty} \frac{\sin w x}{w} \exp \left(-\kappa^{2} w^{2} t\right) d w \\
& =1-\operatorname{erf}\left(\frac{x}{2 \kappa \sqrt{t}}\right)
\end{aligned}
$$

where the function $\operatorname{erf}($.$) denotes the Gaussian error function.$

Let's give the behavior of the temperature distribution of the material in some configurations. See in figures 2,3,4 and 5 the behavior of the temperature distribution of the material $u$ in different cases. The figure 2 describes the behavior of the temperature distribution of the material in the diffusion equation $(\alpha \rightarrow 1)$ when $x$ and $t$ take different values with the diffusion coefficient for the density of the diffusion material fixed to $\kappa^{2}=0.85 .10^{-4} \mathrm{~m}^{2} / \mathrm{s}$ (Hydrogen ion diffusion coefficient). The figure 3 describes the behavior of the temperature distribution of the material in the diffusion equation $(\alpha \rightarrow 1)$ when $x$ takes different values and $t \rightarrow \infty$ and with the diffusion coefficient for the density of the diffusion material fixed to $\kappa^{2}=0.85 .10^{-4} m^{2} / s$. We can observe all the curves decay rapidly. Thus the diffusion becomes more than more rapid. The figure 4 describes the behavior of the temperature distribution of the material in the diffusion equation $(\alpha \rightarrow 1)$ when 


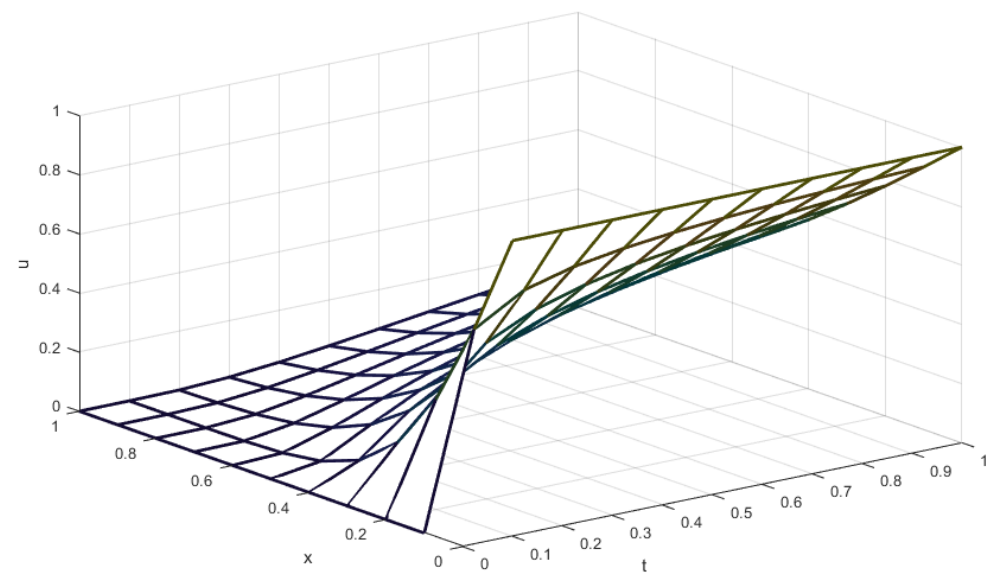

FigurE 2. Surface of the temperature distribution for $\alpha \rightarrow 1, \kappa^{2}=0.85 .10^{-4} \mathrm{~m}^{2} / \mathrm{s}$

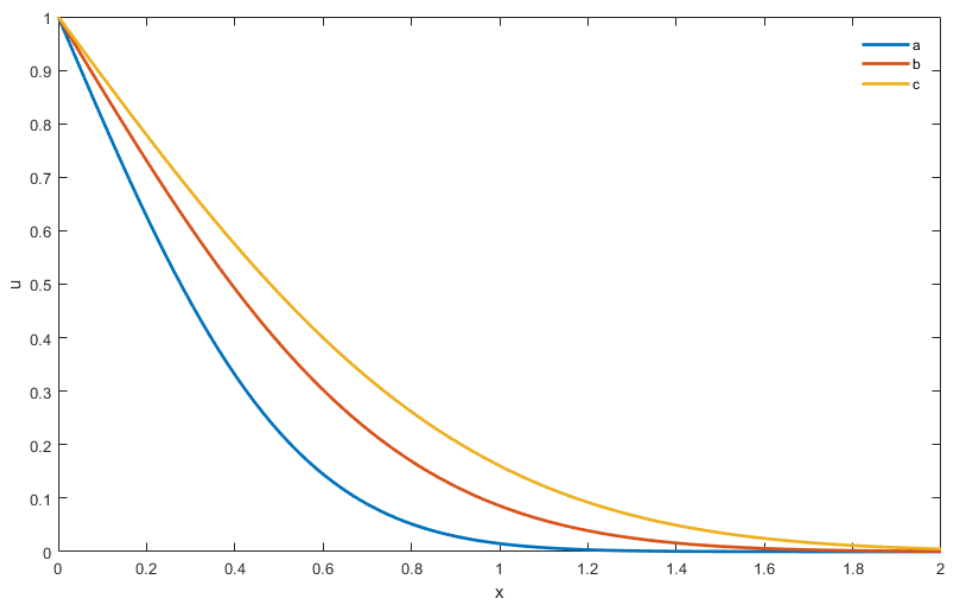

FiguRE 3. The temperatures distributions for $\alpha \rightarrow 1, \kappa^{2}=0.85 .10^{-4} \mathrm{~m}^{2} / \mathrm{s}$ and $t \rightarrow \infty$

successively $x=0.0050, x=0.0065$ and $x=0.0095$ and $t$ takes various values with the diffusion coefficient for the density of the diffusion material fixed to $\kappa^{2}=0.85 .10^{-4} m^{2} / s$. We observe when $x \rightarrow 0$ then the temperature distribution of the material in the diffusion equation $(\alpha \rightarrow 1)$ converge to 1. Furthermore, we can observe all the curves increase slowly. Thus the diffusion is in general very slow. The figure 5 describes the behavior of the temperature distribution of the material in the diffusion equation $(\alpha \rightarrow 1)$ when successively $t=100, t=150$ and $t=200$ and $x$ takes various values with the diffusion coefficient for the density of the diffusion material fixed to $\kappa^{2}=0.85 .10^{-4} \mathrm{~m}^{2} / \mathrm{s}$. We can observe all the curves decay very rapidly. . 


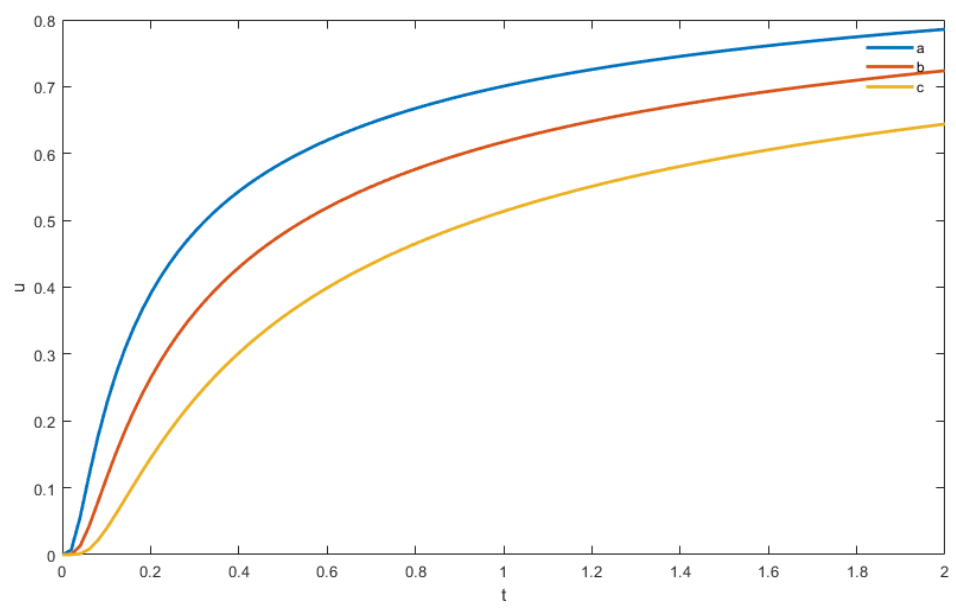

FiguRE 4. The temperatures distributions for $\alpha \rightarrow 1, \kappa^{2}=0.85 .10^{-4} m^{2} / s, x=0.0050 ; 0.0065 ; 0.0095$

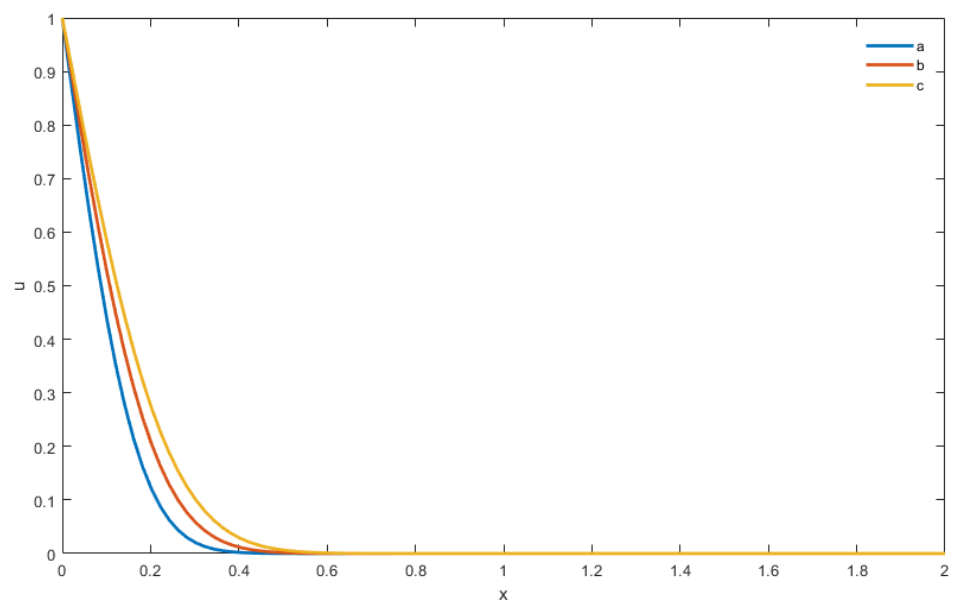

Figure 5. The temperatures distributions for $\alpha \rightarrow 1, \kappa^{2}=0.85 .10^{-4} \mathrm{~m}^{2} / \mathrm{s}, t=100 ; 150 ; 200$

We use a bode plot to interpret the result of this paper graphically. To this end, we use the transfer function given here by the Laplace transform. To reach our conclusion, we compute the capacity

$$
H(s)=\frac{2 \kappa^{2}}{\pi s\left(s+\kappa^{2} w^{2}\right)}
$$

using Matlab code, we obtain the behavior of the amplitude and the phase of the temperature distribution, see in figure 6. 


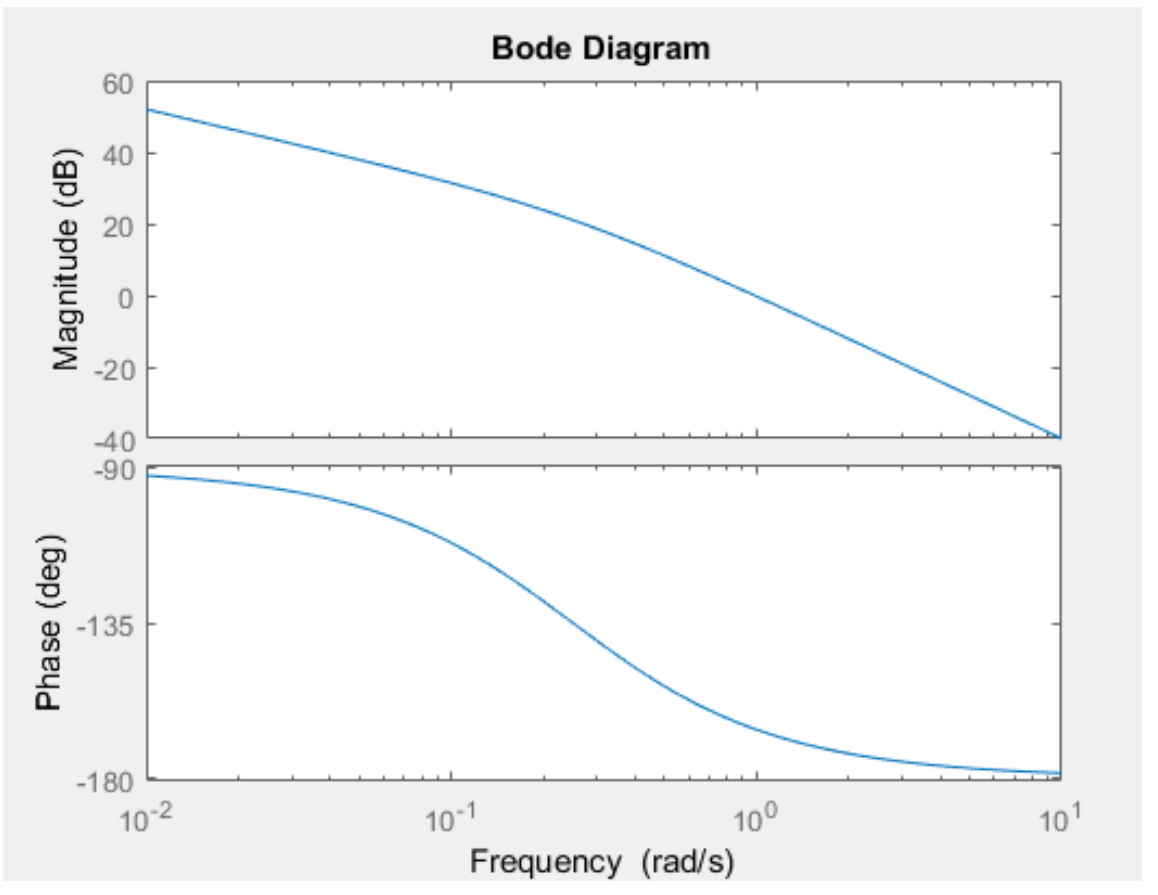

Figure 6. Bode plot of temperature distribution with $\alpha \rightarrow 1, \kappa=0.85 .10^{-4} \mathrm{~m}^{2} / \mathrm{s}$

\section{Analytical Solution of the Fractional Diffusion Equation in Two Dimensional Space}

In this section, we investigate to find the analytical solution of the fractional diffusion equation in twodimensional space expressed as follows

$$
D_{\alpha}^{c} u(x, y, t)=\kappa^{2}\left\{\frac{\partial^{2} u(x, y, t)}{\partial x^{2}}+\frac{\partial^{2} u(x, y, t)}{\partial y^{2}}\right\}
$$

with Dirichlet boundary conditions defined as

• • $u(x, y, 0)=0$ for $x, y>0$,

• $\bullet u(0, y, t)=u(x, 0, t)=1$ for $t>0$.

We repeat the same reasoning as in Section 3. We apply the Fourier sine transform. Multiplying equation (4.1) by $\frac{2}{\pi} \sin w x \sin \eta y$ and integrating it between 0 to $\infty$ successively respecting $x$ and $y$, we get that:

$$
\begin{aligned}
& D_{\alpha}^{c} u_{s}(w, \eta, t)=\kappa^{2}\left\{\frac{2\left(w^{2}+\eta^{2}\right)}{\pi w \eta} u_{s}(0, t)-\left(w^{2}+\eta^{2}\right) u_{s}(w, \eta, t)\right\} \\
& D_{\alpha}^{c} u_{s}(w, \eta, t)=\frac{2 \kappa^{2}\left(w^{2}+\eta^{2}\right)}{\pi w \eta}-\kappa^{2}\left(w^{2}+\eta^{2}\right) u_{s}(w, \eta, t) .
\end{aligned}
$$

where $u_{s}(w, \eta, t)$ denotes the Fourier sine transform of $u(x, y, t)$. Rearranging, we obtain the following fractional differential equation defined as

$$
D_{\alpha}^{c} u_{s}(w, \eta, t)+\kappa^{2}\left(w^{2}+\eta^{2}\right) u_{s}(w, \eta, t)=\frac{2 \kappa^{2}\left(w^{2}+\eta^{2}\right)}{\pi w \eta} .
$$


We apply the Laplace transform to both sides of equation (4.2). We obtain the following relationships

$$
\begin{aligned}
s^{\alpha} \bar{u}_{s}(w, \eta, s)+\kappa^{2}\left(w^{2}+\eta^{2}\right) \bar{u}_{s}(w, \eta, t) & =\frac{2 \kappa^{2}\left(w^{2}+\eta^{2}\right)}{\pi w \eta s} \\
\bar{u}_{s}(w, \eta, s) & =\frac{2 \kappa^{2}\left(w^{2}+\eta^{2}\right)}{\pi w \eta s\left(s^{\alpha}+\kappa^{2}\left(w^{2}+\eta^{2}\right)\right)} .
\end{aligned}
$$

where $\bar{u}_{s}(w, \eta, s)$ denotes the Laplace transform of $u_{s}(w, \eta, t)$. To obtain the analytical solution of the fractional diffusion equation (4.1), we rewrite the Laplace transform (4.3) as follows

$$
\bar{u}_{s}(w, \eta, s)=\frac{2}{\pi w \eta}\left\{\frac{1}{s}-\frac{s^{\alpha-1}}{s^{\alpha}+\kappa^{2}\left(w^{2}+\eta^{2}\right)}\right\} .
$$

Finally, to get the analytical solution of the fractional diffusion equation (4.1), we apply the inverse of Laplace transform to both sides of equation (4.4) and the inverse of Fourier sine transform on the obtained equation. We get

$$
u(x, y, t)=\frac{4}{\pi^{2}} \int_{0}^{\infty} \frac{\sin w x}{w} \int_{0}^{\infty} \frac{\sin \eta y}{y}\left\{1-E_{\alpha}\left(-\kappa^{2}\left(w^{2}+\eta^{2}\right) t^{\alpha}\right)\right\} d \eta d w .
$$

We investigate the analytical solution of the diffusion equation in two-dimensional space obtained when $\alpha \rightarrow 1$. To this end, we pick the Laplace transform function defined to equation (4.4) when $\alpha \rightarrow 1$, defined by

$$
\bar{u}_{s}(w, \eta, s)=\frac{2}{\pi w \eta}\left\{\frac{1}{s}-\frac{1}{s+\kappa^{2}\left(w^{2}+\eta^{2}\right)}\right\} .
$$

Applying the inverse of Laplace transform and the inverse of the Fourier sine transform, we obtain that

$$
\begin{aligned}
u(x, y, t) & =\frac{4}{\pi^{2}} \int_{0}^{\infty} \frac{\sin w x}{w} \int_{0}^{\infty} \frac{\sin \eta y}{y}\left\{1-\exp \left(-\kappa^{2}\left(w^{2}+\eta^{2}\right) t\right)\right\} d \eta d w \\
& =1-\frac{4}{\pi^{2}} \int_{0}^{\infty} \frac{\sin w x}{w} \int_{0}^{\infty} \frac{\sin \eta y}{y} \exp \left(-\kappa^{2}\left(w^{2}+\eta^{2}\right) t\right\} d \eta d w
\end{aligned}
$$

We use the Gaussian error function $\operatorname{erf}($.$) , we obtain the following form$

$$
u(x, y, t)=1-\operatorname{erf}\left(\frac{x}{2 \kappa \sqrt{t}}\right) \operatorname{erf}\left(\frac{y}{2 \kappa \sqrt{t}}\right) .
$$

That is the analytical solution of the diffusion equation in two-dimensional space obtained when $\alpha \rightarrow 1$.

Let's give the behavior of the temperature distribution of the material in the diffusion equation in some configurations. Figure 7 describes the behavior of the temperature distribution of the material in the diffusion equation in two-dimensional space when $x=y$ and $t$ takes various values. Figure 8 describes the behavior of the temperature distribution of the material in the diffusion equation in two-dimensional space when $x=y$ and $t \rightarrow \infty$, we observe the Gaussian profile of the temperature distribution of the material in the diffusion equation. Figure 9 describes the behavior of the temperature distribution of the material in the diffusion equation in two-dimensional space when $x=y \rightarrow 0$ and $t$ take various values. We observe all curves increase rapidly. 


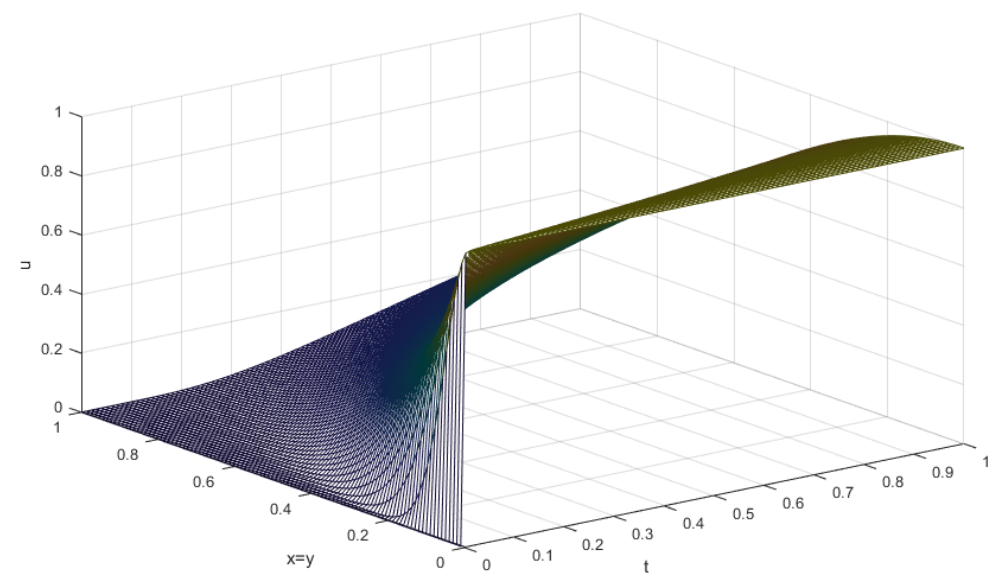

FiguRE 7 . Surface of the temperature distribution of the material in the diffusion equation for $\alpha \rightarrow 1, \kappa=0.85 .10^{-4} \mathrm{~m}^{2} / \mathrm{s}, x=y$

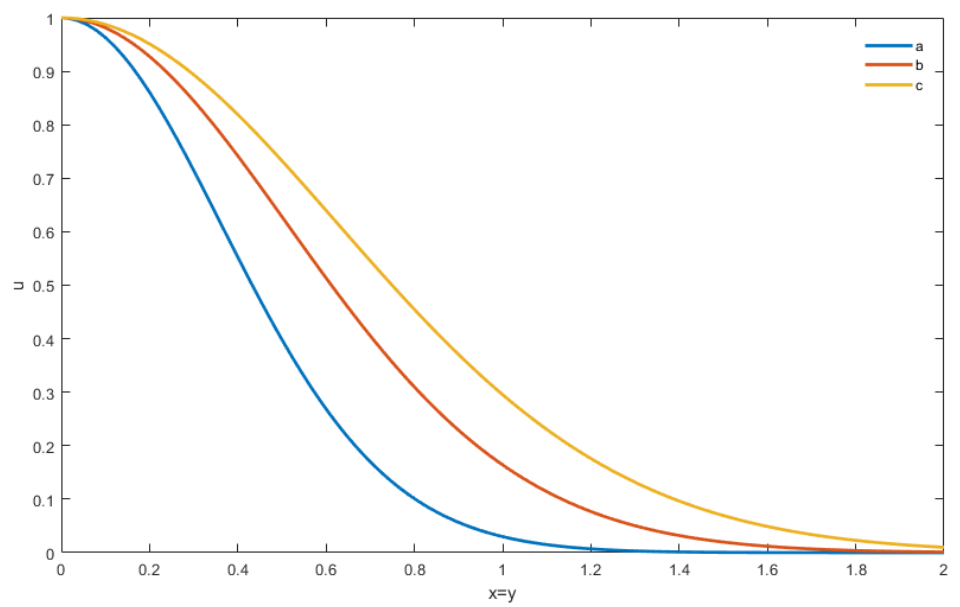

Figure 8. Temperature distribution of the material in the diffusion equation for $\alpha \rightarrow 1$, $\kappa=0.85 .10^{-4} m^{2} / s, x=y$ and $t \rightarrow \infty$

\section{Analytical Solution of the Cattaneo-Hristov Diffusion Equation}

Hristov in [12] [13], stating with Cattaneo constructive relaxation with Jeffrey's kernel proposes a new elastic heat diffusion equation described by the Caputo-Fabrizio fractional derivative. Diffusion phenomena, of heat or mass, are generally explained as a consequence of the conservative law by the relationships [12]

$$
\rho C_{p} \frac{\partial T}{\partial t}=-\frac{\partial q}{\partial x} ; \quad q(x, t)=-k \frac{\partial T(x, t)}{\partial x} \Rightarrow \rho C_{p} \frac{\partial T}{\partial t}=k \frac{\partial^{2} T}{\partial x^{2}}
$$

where the flux of heat is given by the following relationship

$$
q(x, t)=-\int_{-\infty}^{t} R(x, t) \nabla T(x, t-s) d s
$$




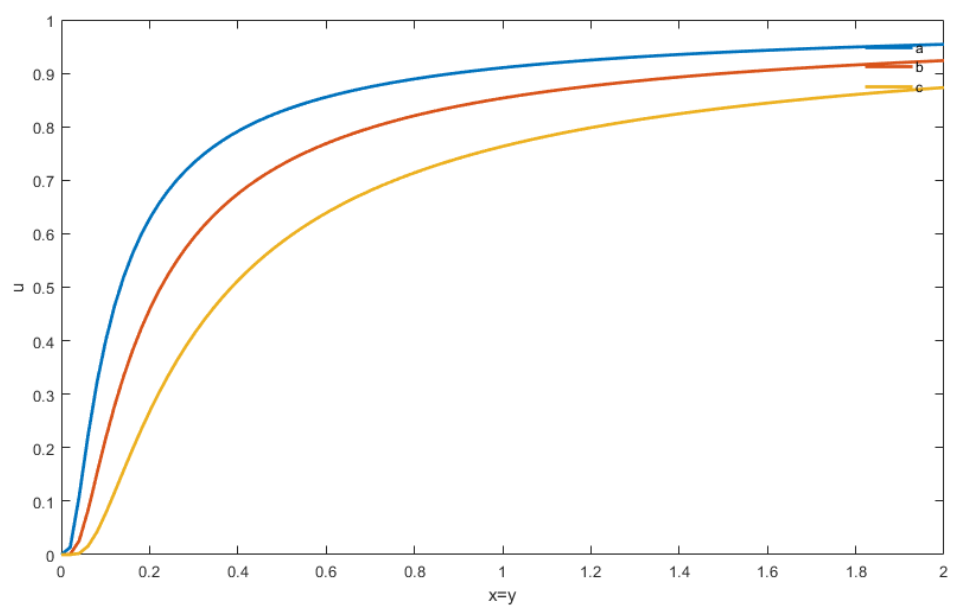

Figure 9. Temperature distribution for $\alpha=\rightarrow 1, \kappa^{2}=0.85 .10^{-4} m^{2} / s, x=y \rightarrow 0$ and $t$

In this case of space independent damping the function $R(x, t)$ it can be represented by the Jeffrey kernel $R(t)=\exp (-(t-s) / \tau)$ where $\tau$ designs a finite relaxation term [12] [26]. Continuing the constructive equations, the energy balance yields the Cattaneo equation defined as the following form [12]

$$
\frac{\partial T(x, t)}{\partial t}=-\frac{k_{2}}{\tau \rho C_{p}} \int_{0}^{t} \exp (-(t-s) / \tau) \frac{\partial T(x, s)}{\partial x} d s
$$

With equation (5.3), the energy conservative equation of the internal energy result in the Jeffrey type interodifferential equation [12] in the form

$$
\frac{\partial T(x, t)}{\partial t}=\frac{k_{1}}{\rho C_{p}} \frac{\partial^{2} T(x, t)}{\partial x^{2}}+\frac{k_{2}}{\tau \rho C_{p}} \int_{-\infty}^{t} \exp (-(t-s) / \tau) \frac{\partial^{2} T(x, s)}{\partial x^{2}} d s .
$$

Finally, using the concept of the Caputo-Fabrizio fractional derivative recently introduced in [6] and some assumptions, see more details in [6], Hristov arrives to the complete Cattaneo-Hristov diffusion equation [12] [13] expressed as the following form

$$
\frac{\partial T(x, t)}{\partial t}=a_{1} \frac{\partial^{2} T(x, t)}{\partial x^{2}}+a_{2}(1-\alpha)_{0}^{C F} D_{t}^{\alpha}\left(\frac{\partial^{2} T(x, t)}{\partial x^{2}}\right) .
$$

where $a_{1}=\frac{k_{1}}{\rho C_{p}}$ and $a_{2}=\frac{k_{2}}{\rho C_{p}}$ with $\rho=$ const, $C_{p}=$ const. The constant $k_{1}$ and $k_{2}$ represent successively the effective thermal conductivity and the elastic conductivity. ${ }_{0}^{C F} D_{t}^{\alpha}$ denotes the Caputo-Fabrizio fractional derivative, see in [6] and $T$ represents the temperature distribution. The Dirichlet boundary conditions considered in this paper is defined in the following form

$\bullet \bullet T(x, 0)=0$ for $x>0$,

$\bullet \bullet T(0, t)=1$ for $t>0$.

The equation (5.5) is known as the entire Cattaneo-Hristov equation of transition heat diffusion equation. The Cattaneo-Hristov diffusion equation allows in-depth investigations of the role of the damping kernel on 
the behavior of the heat diffusion process and the telegraph equation [1]. The second term of the CattaneoHristov equation

$$
\frac{\partial T(x, t)}{\partial t}=a_{2}(1-\alpha)_{0}^{C F} D_{t}^{\alpha}\left(\frac{\partial^{2} T(x, t)}{\partial x^{2}}\right)
$$

is known as the elastic part of the heat diffusion equation process and was subject of investigations done by Koca et al. in [26]. In [26] Koca et al. propose analytical and numerical solutions of the elastic part of the heat diffusion equation process described by the Caputo-Fabrizio fractional derivative. In [12], Hristov proposes an approximation of the solution using an integral method based on a finite penetration depth.

In this section, we investigate to find the analytical solution of the complete Cattaneo-Hristov diffusion equation (5.5). The boundary conditions considered in this paper are particular cases which we can obtain with Cattaneo-Hristov model of diffusion. And all results found in this section can be modified when the boundary conditions change. The method of the resolution used in the previous section to get the analytical solution of the fractional diffusion equations in one and two-dimensional spaces doesn't change. Before applying the Fourier sine transform and the Laplace transform, we recall the Laplace transform of the Caputo-Fabrizio fractional derivative given by

$$
\mathcal{L}\left\{{ }_{0}^{C F} D_{t}^{\alpha} f(t)\right\}=\frac{s \mathcal{L}\{f(t)\}(s)-f(0)}{s+\alpha(1-s)} .
$$

To get the analytical solution of the complete Cattaneo-Hristov diffusion equation, we multiply equation (5.5) by $\frac{2}{\pi} \sin w x$ and integrating it between 0 to $\infty$; we obtain the following differential equation

$$
\begin{aligned}
\frac{\partial T_{s}(w, t)}{\partial t} & =a_{1}\left\{\frac{2}{\pi} w-w^{2} T_{s}(w, t)\right\}+a_{2}(1-\alpha)_{0}^{C F} D_{t}^{\alpha}\left\{\frac{2}{\pi} w-w^{2} T_{s}(w, t)\right\} \\
& =a_{1}\left\{\frac{2}{\pi} w-w^{2} T_{s}(w, t)\right\}-a_{2} w^{2}(1-\alpha)_{0}^{C F} D_{t}^{\alpha} T_{s}(w, t) .
\end{aligned}
$$

where $T_{s}(w, t)$ denotes the Fourier sine transform of $T(x, t)$. Applying the Laplace transform to both sides of equation (5.8), we get that

$$
\bar{T}_{s}(w, t)=\frac{2 a_{1} w(\alpha+(1-\alpha) s)}{\pi s\left\{(1-\alpha) s^{2}+\left(\alpha+(1-\alpha)\left(a_{1} w^{2}+a_{2} w^{2}\right)\right) s+a_{1} w^{2} \alpha\right\}} .
$$

where $\bar{T}_{s}(w, t)$ denotes the Laplace transform of $T_{s}(w, t)$. Let that $\lambda=\frac{\alpha}{1-\alpha}$ with $\alpha \neq 1$ and then equation (5.9) can be rewritten as follows

$$
\bar{T}_{s}(w, t)=\frac{2 a_{1} w(\lambda+s)}{\pi s\left\{s^{2}+\left(\lambda+\left(a_{1} w^{2}+a_{2} w^{2}\right)\right) s+a_{1} w^{2} \lambda\right\}} .
$$

The transformation (5.10) is essential in a sense we use it for every specific order. The equation (5.10) can be rewritten as a series, and then we obtain the following relationships

$$
\bar{T}_{s}(w, t)=\frac{2}{\pi} \sum_{k=0}^{\infty}(-1)^{k}\left(a_{1}\right)^{k} w^{2 k+1} \lambda^{k} \frac{\lambda s^{1-(3+k)}+s^{1-(2+k)}}{\left(s+\left(\lambda+\left(a_{1} w^{2}+a_{2}\right)\right)\right)^{k+1}} .
$$


Let that $\mu=\left(\lambda+\left(a_{1} w^{2}+a_{2} w^{2}\right)\right)$, applying the inverse of Laplace transformation and using Mittag-Leffler functions with three parameters, we get that

$$
T_{s}(w, t)=\frac{2}{\pi} \sum_{k=0}^{\infty} \frac{(-1)^{k}}{k !}\left(a_{1}\right)^{k} w^{2 k+1} \lambda^{k}\left[\lambda t^{2 k+2} E_{1,3+k}^{(k)}(-\mu t)+t^{2 k+1} E_{1,2+k}^{(k)}(-\mu t)\right] .
$$

Finally, we get the analytical solution of the Cattaneo-Hristov diffusion equation, by applying the inverse of Fourier sine transform

$$
\begin{aligned}
T(x, t) & =\frac{2 a_{1}}{\pi} \int_{0}^{\infty} w \sin (w x) \sum_{k=0}^{\infty} \frac{(-1)^{k}}{k !}\left(a_{1}\right)^{k} w^{2 k} \lambda^{k} \\
& \times\left[\lambda t^{2 k+2} E_{1,3+k}^{(k)}(-\mu t)+t^{2 k+1} E_{1,2+k}^{(k)}(-\mu t)\right] d w .
\end{aligned}
$$

As in the previous section, we analyze the particular case of the Cattaneo-Hristov diffusion equation obtained when $\alpha \rightarrow 1$. The Laplace transform is given using the equation (5.10) by

$$
\bar{T}_{s}(w, t)=\frac{2}{\pi} \frac{1}{w}\left\{\frac{1}{s}-\frac{1}{s+a_{1} w^{2}}\right\} .
$$

Applying the inverse of Laplace transform to both sides to equation (5.14) and the inverse of Fourier sine transform we get

$$
\begin{aligned}
T(x, t) & =\frac{2}{\pi} \int_{0}^{\infty} \frac{\sin (w x)}{w}\left\{1-\exp \left(-a_{1} w^{2} t\right)\right\} d w \\
& =1-\frac{2}{\pi} \int_{0}^{\infty} \frac{\sin (w x)}{w} \exp \left(-a_{1} w^{2} t\right) d w \\
& =1-\operatorname{erf}\left(\frac{x}{2 \sqrt{a_{1} t}}\right) .
\end{aligned}
$$

One can observe this solution is similar to the solution obtained in the classical diffusion equation. Thus the surface described by the solution of the particular Cattaneo-Hristov diffusion equation considered above is identical to the surface represented by the solution of the classical diffusion equation.

\section{Conclusion}

The complete Cattaneo-Hristov equation of the transient heat diffusion equation introduced by Hristov was considered in this paper. The problems opened by Hristov with this new constructive equation in the fractional diffusion equation are the problem consisting of getting the numerical solutions, the problem consisting of finding the analytical solutions and the problem consisting to get an approximate solutions. Hristov proposes an estimate for the solution of the Cattaneo-Hristov diffusion equation using a finite penetration depth, Koca and Atangana in their works suggest the analytical and the numerical solutions of the elastic part of the heat diffusion equation process. The numerical solution of the complete Cattaneo-Hristov equation of the transient heat diffusion equation was considered in recent works done by Alkahtani and Atangana. This paper proposes the analytical solution of the complete Cattaneo-Hristov equation of the transient heat diffusion equation. The integral method used in the resolution combines both the Fourier sine transform and 
the Laplace transform. This paper offers a useful analytical solution of the fractional diffusion equation in two-dimensional space. Some special cases of the fractional diffusion equations were discussed and illustrated graphically.

\section{REFERENCES}

[1] B. S. T. Alkahtani and A. Atangana. A note on cattaneo-hristov model with non-singular fading memory. Therm. Sci., 21(1)(2017), 1-7.

[2] A. Atangana and D. Baleanu. New fractional derivatives with nonlocal and non-singular kernel: theory and application to heat transfer model. arXiv preprint arXiv:1602.03408, (2016).

[3] A. Atangana and JF. Gomez-Aguilar. Fractional derivatives with noindex law property: Application to chaos and statistics. Chaos Solitons Fractals, 114 (2018), 516-535.

[4] A. Atangana and I. Koca. Chaos in a simple nonlinear system with atanganabaleanu derivatives with fractional order. Chaos Solitons Fractals, 89 (2016), 447-454.

[5] L. Beghin. Fractional diffusion-type equations with exponential and logarithmic differential operators. Stoc. Proc. Appl., $128(7)(2018), 2427-2447$.

[6] M. Caputo and M. Fabrizio. A new definition of fractional derivative without singular kernel. Progr. Fract. Differ. Appl., $1(2)(2015), 1-13$.

[7] A. C. Escamilla, JF. G. Aguilar, L. Torres, and RF. E. Jimnez. A numerical solution for a variable-order reactiondiffusion model by using fractional derivatives with non-local and non-singular kernel. Phys. A: Stat. Mech. Appl., 491(2018), 406-424

[8] H. Delavari, D. Baleanu, and J. Sadati. Stability analysis of caputo fractional-order nonlinear systems revisited. Nonlinear Dyn., 67(4) (2012), 2433-2439.

[9] E. F. D. Goufo. Chaotic processes using the two-parameter derivative with non-singular and non-local kernel: basic theory and applications. Chaos: An Inter. J. Nonlinear Sci., 26(8) (2016), 084-305.

[10] J. Hristov. On the atangana-baleanu derivative and its relation to the fading memory concept: The diffusion equation formulation. Trends in theory and applications of fractional derivatives with Mittag-Leffler kernel, Springer. 2019.

[11] J. Hristov. Approximate solutions to fractional subdiffusion equations. Eur. Phys. J. Spect. Topics, 193(1)(2011), $229-243$.

[12] J. Hristov. Transient heat diffusion with a non-singular fading memory: from the cattaneo constitutive equation with jeffrey's kernel to the caputo-fabrizio time-fractional derivative. Therm. Sci., 20(2) (2016), 757-762.

[13] J. Hristov. Derivation of the fractional dodson equation and beyond: Transient diffusion with a non-singular memory and exponentially fadingout diffusivity. Progr. Fract. Differ. Appl, 3(4) (2017), 1-16.

[14] J. Hristov. Multiple integral-balance method basic idea and an example with mullins model of thermal grooving. Therm. Sci., 21(2017), 1555-1560.

[15] J. Hristov. The non-linear dodson diffusion equation: Approximate solutions and beyond with formalistic fractionalization. Math. Nat. Sci., 1(1) (2017), 1-17.

[16] J. Hristov. Fourth-order fractional diffusion model of thermal grooving: integral approach to approximate closed form solution of the mullins model. Math. Model. Nat. Phenom. 13(1)(2018), 6.

[17] J. Hristov. Integral-balance solution to nonlinear subdiffusion equation. Front. Fract. Calcu., 1(2018), 70.

[18] J. Hristov. The heat radiation diffusion equation: Explicit analytical solutions by improved integral-balance method. Therm. Sci., 22(2) (2018), 777-788. 
[19] J. Hristov. Integral balance approach to 1-d space-fractional diffusion models. Math. Meth. Eng., (2019), 111-131, Springer.

[20] J. Hristov. A transient flow of a non-newtonian fluid modelled by a mixed time-space derivative: An improved integralbalance approach. Math. Meth. Eng., (2019), 153-174, Springer.

[21] F. Jarad and T. Abdeljawad. A modified laplace transform for certain generalized fractional operators. Res. Nonlinear Anal., (2)(2018), 88-98.

[22] F. Jarad, E. Ugurlu, T. Abdeljawad, and Dumitru Baleanu. On a new class of fractional operators. Adv. Diff. Equa., (1)(2017), 247.

[23] H. Jordan. Steady-state heat conduction in a medium with spatial non-singular fading memory derivation of caputo-fabrizio spacefractional derivative from cattaneo concept with jeffrey's kernel and analytical solutions. Therm. Sci., 21(2) (2017), 827-839.

[24] U. N. Katugampola. A new approach to generalized fractional derivatives. Bull. Math. Anal. Appl, 6(4)(2014), 115.

[25] A. A. Kilbas, M Rivero, L Rodriguez-Germa, and JJ Trujillo. Caputo linear fractional differential equations. IFAC Proc. 39(11) (2006), 52-57.

[26] I. Koca and Abdon Atangana. Solutions of cattaneo-hristov model of elastic heat diffusion with caputo-fabrizio and atangana-baleanu fractional derivatives. Therm. Sci., 21 (2017), 2299-2305.

[27] L. Li, J. G. Liu, and L. Wang. Cauchy problems for kellersegel type timespace fractional diffusion equation. J. Differ. Equ., $265(3)(2018)$, 1044-1096.

[28] Y. Li, Y. Q. Chen, and I. Podlubny. Mittagleffler stability of fractional order nonlinear dynamic systems. Auto., 45(8) (2009), 1965-1969.

[29] Y. Li, F. Liu, I. W. Turner, and T. Li. Time-fractional diffusion equation for signal smoothing. Appl. Math. Comp., 326 (2018), 108116.

[30] J. Losada and J. J. Nieto. Properties of a new fractional derivative without singular kernel. Progr. Fract. Differ. Appl, $1(2)(2015), 87-92$.

[31] Y. Ma, F. Zhang, and C. Li. The asymptotics of the solutions to the anomalous diffusion equations. Comput. Math. Appl., 66(5)(2013), 682-692.

[32] T. Myers. Optimal exponent heat balance and refined integral methods applied to stefan problems. Int. J. Heat Mass Transfer, 53(5-6) (2010), 1119-1127.

[33] K. M. Owolabi and A. Atangana. Robustness of fractional difference schemes via the caputo subdiffusion-reaction equations. Chaos, Solitons Fractals, 111 (2018), 119-127.

[34] I. Podlubny. Fractional differential equations: an introduction to fractional derivatives, fractional differential equations, to methods of their solution and some of their applications, (1998), 198. Acad. Press.

[35] I. Podlubny. Matrix approach to discrete fractional calculus ii: Partial fractional differential equations. (2009).

[36] S. Priyadharsini. Stability of fractional neutral and integrodifferential systems. J. Fract. Calc. Appl.,7(1) (2016), 87-102.

[37] Z. Ruan, W. Zhang, and Zewen Wang. Simultaneous inversion of the fractional order and the space-dependent source term for the time-fractional diffusion equation. Appl. Math. Comput., 328 (2018), 365-379.

[38] K. M. Saad, D. Baleanu, and A. Atangana. New fractional derivatives applied to the kortewegde vries and korteweg-de vries-burgers equations. Comput. Appl. Math., 37 (2018), 52035216.

[39] Y. Salehi, M. T. Darvishi, and W. E. Schiesser. Numerical solution of space fractional diffusion equation by the method of lines and splines. Appl. Math. Comput., 336 (2018), 465-480.

[40] N. Sene. Exponential form for lyapunov function and stability analysis of the fractional differential equations. J. Math. Comput. Sci. 18(4)(2018), 388-397. 
[41] N. Sene. Lyapunov characterization of the fractional nonlinear systems with exogenous input. Fractal Fract., (2018), 2(2):17.

[42] N. Sene. Solutions for some conformable differential equations. Progr. Fract. Differ. Appl., 4(4)(2018), 493-501.

[43] N. Sene. Stokes first problem for heated flat plate with atangana-baleanu fractional derivative. Chaos Soli. Fract., 117 (2018), 68-75.

[44] S. Shen, F. Liu, and V. V. Anh. The analytical solution and numerical solutions for a two-dimensional multi-term time fractional diffusion and diffusion-wave equation. J. Comput. Appl. Math., 345 (2019), 515-534.

[45] J. Zhang, X. Zhang, and B. Yang. An approximation scheme for the time fractional convectiondiffusion equation. Appl. Math. Comput., 335(2018), 305-312. 